\title{
Optical conductivity of colossal magnetorestistance compounds: Role of orbital degeneracy in the ferromagnetic phase
}

\author{
Peter Horsch ${ }^{a}$, Janez Jaklic ${ }^{a, b, *}$, and Frank Mack ${ }^{a}$ \\ (a) Max-Planck-Institut für Festkörperforschung, Heisenbergstr. 1, D-70569 Stuttgart (Germany) \\ (b) Max-Planck-Institut für Physik komplexer Systeme, Nöthnitzer Str. 38, D-01187 Dresden (Germany)
}

(April 17, 1998)

\begin{abstract}
Recent optical conductivity $\sigma(\omega)$ experiments have revealed an anomalous spectral distribution in the ferromagnetic phase of the perovskite system $\mathrm{La}_{1-x} \mathrm{Sr}_{x} \mathrm{MnO}_{3}$. Using finite temperature diagonalization techniques we investigate $\sigma(\omega)$ for a model that contains only the $e_{g}$-orbital degrees of freedom. Due to strong correlations the orbital model appears as a generalized $t$ - $J$ model with anisotopic interactions and 3-site hopping. In the orbital $t$ - $J$ model $\sigma(\omega)$ is characterized by a broad incoherent spectrum with increasing intensity as temperature is lowered, and a Drude peak with small weight, consistent with experiment. Our calculations for two-dimensional systems, which may have some particular relevance for the double-layer manganites, show that the scattering from orbital fluctuations can explain the order of magnitude of the incoherent part of $\sigma(\omega)$ in the low temperature ferromagnetic phase. Moreover orbital correlation functions are studied and it is shown that $x^{2}-y^{2}$ orbital order is prefered in the doped planar model at low temperature.
\end{abstract}

PACS numbers: 75.10.-b 75.40.Cx 75.40.Gb 25.40.Fq

\section{INTRODUCTION}

The physics of doped manganese-oxide ceramics (manganites) is characterized by strong correlations and the complex interplay of spin-, charge-, and orbital-degrees of freedom as well as the coupling to the lattice, e.g. via Jahn-Teller coupling [1,2]. This complexity is directly evident from the large number of phases in a typical phase diagram. To quantify the different mechanisms it is helpful to analyze experiments where for certain parameters one or the other degree of freedom is frozen out. Important experiments in this respect are the very detailed investigations of the optical conductivity of $\mathrm{La}_{1-x} \mathrm{Sr}_{x} \mathrm{MnO}_{3}$ by Okimoto et al. [3]. These experiments show (a) a pseudogap in $\sigma(\omega)$ for temperatures above the Curie temperature $T_{c}$ (paramagnetic phase) with $\sigma(\omega)$ essentially linear in $\omega$, and (b) the evolution of a broad incoherent distribution in the range $0 \leq \omega<1.0$ $\mathrm{eV}$ below $T_{c}$, which still grows at temperatures below $T_{c} / 10$, where the magnetization is already close to saturation. Such a temperature dependence of $\sigma(\omega)$ over a wide energy region is quite unusual as compared with other strongly correlated electron systems near a metalinsulator phase boundary [3]. Interestingly there is in addition a narrow Drude peak with width of about 0.02 $\mathrm{eV}$ and little weight superimposed to the broad incoherent spectrum. In view of the large energy scale of $1 \mathrm{eV}$ it is plausible that the orbital degeneracy is the source of this incoherent motion, since the spin degrees of freedom are essentially frozen out.

Further optical studies for various 3D manganites [5 7 ] as well as for the bilayer system $\mathrm{La}_{1.2} \mathrm{Sr}_{1.8} \mathrm{Mn}_{2} \mathrm{O}_{7}[8]$ confirm the presence of the large incoherent absorption in the ferromagnetic state.

Although the importance of this experiment was recognized immediately, the few theoretical studies 99 11] were confined to simplified models or approximations, thereby ignoring important aspects of the full many body problem.

The aim of this work is to show that in a model which accounts for the orbital degeneracy, yet assumes that the spins are fully polarized, the broad incoherent spectral distribution of $\sigma(\omega)$, its increase with decreasing temperature, as well as the order of magnitude of $\sigma(\omega)$ at small $\omega$ can be explained. Our calculation also accounts for a small and narrow Drude peak as observed by Okimoto et al. in the range $\omega<0.05 \mathrm{eV}$. This is a clear indication of coherent motion of charge carriers with small spectral weight, i.e. in a model where due to the orbital degeneracy incoherent motion is dominant.

The colossal magnetoresistance (CMR) of manganites 12. and their transport properties are usually studied on the basis of the double exchange (DE) Hamiltonian or the more general ferromagnetic Kondo lattice model (KLM) 13. The essence of these models is the high-spin configuration of $e_{g}$-electron and $t_{2 g}$-core electron spins due to a strong ferromagnetic Kondo exchange interaction $K \sim 1 \mathrm{eV}$. The kinetic energy in the partially filled $e_{g}$ band is lowered when neighboring spin are aligned leading to a low-temperature ferromagnetic phase, while the high- $T$ paramagnetic phase is disordered with a high resistivity. Although this model provides an explanation of the phases necessary for a qualitative understanding of CMR, it has been stressed that the double exchange mechanism is not sufficient for a quantitative description [14.

Furthermore we would like to stress here, that the KLM without orbital degeneracy also fails to explain the broad incoherent $\sigma(\omega)$ spectra observed in the ferromagnetic phase as $T \rightarrow 0$ [3, 价. The KLM would instead lead to a sharp Drude absorption because of the ferromagnetic alignement [15]. This underlines the importance of 
the $e_{g}$ orbital degeneracy, although there are alternative proposals invoking strong electron-phonon coupling and lattice polaron formation [1, 14, 16].

We start our discussion with a generic Hamiltonian for the manganite systems, i.e. the ferromagnetic Kondo lattice model with degenerate $e_{g}$-orbitals, and derive for the spin-polarized case an effective model which contains only the orbital degrees of freedom. This orbital model consists of a hopping term between the same and different orbitals $\alpha$ and $\beta$ on neighbor sites and an orbital interaction. Renaming $\alpha=\sigma$ where $\sigma=\uparrow$ or $\downarrow$ the model maps on a generalized anisotropic $t$ - $J$ model. The usual $t-J$ model known from the cuprates appears as a special case. Our derivation includes the 3-site hopping processes, which appear as a natural consequence of the strong coupling limit. Although such terms do not influence the orbital order for integer filling, they are important for the proper description of transport properties in the doped systems [17 20].

Because of the complexity of the orbital model we present here first a numerical study of the orbital correlations and of the frequency dependent conductivity. Although there exist studies of the interplay of orbital and spin order at integer filling for $\mathrm{LaMnO}_{3}$ [21,22], the effect of doping has not been considered so far. The finite temperature diagonalization 23,24] serves here as an unbiased tool to study the optical conductivity and the change of orbital correlations as function of doping and temperature.

The outline of the paper is as follows. In Section II we discuss the system in the frame of ferromagnetic Kondo lattice model with degenerate $e_{g}$-orbitals and derive for the spin-polarized case an effective model which contains only the orbital degrees of freedom. In Section III we present first results for the optical conductivity for this orbital model. Apart from the frequency dependent conductivity spectra $\sigma(\omega)$ we also present the variation of the optical sum rule and the Drude weight with temperature for various doping concentrations. Furthermore we show that 3 -site hopping processes, which appear in a natural way in the strong coupling limit, enhance significantly the Drude weight and the coherent motion of charge carriers. This section also contains a brief discussion of related theoretical work. The evolution of orbital correlations with temperature is discussed in Section IV for the two-dimensional model at different doping concentrations. In particular we allow here for a rotation of the local quantization axis when calculating these correlation functions. The results presented were obtained by a finite temperature diagonalization method for finite clusters with up to $\mathrm{N}=16$ lattice sites. The final Section contains a brief summary.

\section{MODEL}

We start from the generic ferromagnetic Kondo lattice model for the manganites, where the $\mathrm{Mn} e_{g}$-electrons are coupled to core spins $\vec{S}_{\mathbf{i}}$ (formed by the $t_{2 g}$ orbitals) and a local repulsion $U$ between the electrons in the two $e_{g}$ orbitals:

$$
H=H_{\text {band }}+H_{\text {int }}+H_{\text {Kondo }} .
$$

$H_{\text {band }}$ describes the hopping of $e_{g}$-electrons between sites $i$ and $j$. We take the electrons to have two-fold orbital degeneracy labelled by a Roman index (a,b) and two fold spin degeneracy labelled by a Greek index $\left(\sigma, \sigma^{\prime}\right)$. Explicitly,

$$
H_{b a n d}=\sum_{\mathbf{i} a \sigma} E_{\mathbf{i}}^{a} d_{\mathbf{i} a \sigma}^{\dagger} d_{\mathbf{i} a \sigma}+\sum_{\langle\mathbf{i}\rangle a b \sigma}\left(t_{\mathbf{i} \mathbf{j}}^{a b} d_{\mathbf{i} a \sigma}^{\dagger} d_{\mathbf{j} b \sigma}+H . c .\right) .
$$

The hopping matrix element $t_{\mathrm{ij}}^{a b}$ is a real symmetric matrix whose form depends on the choice of basis in $a b$ space and the direction of the $\mathbf{i}-\mathbf{j}$ bond. For the present study we shall use $|x\rangle \sim x^{2}-y^{2}$ and $|z\rangle \sim\left(3 z^{2}-r^{2}\right) / \sqrt{3}$ as basis for the $e_{g}$ orbitals. From the Slater-Koster rules follows 2527]: $\left|t^{x x}\right|=\frac{3}{4} t ;\left|t^{x z}\right|=\frac{\sqrt{3}}{4} t ;\left|t^{z z}\right|=\frac{1}{4} t$ in $\mathrm{x}-$ and y- direction. For a cubic lattice the matrix elements in z-direction are $\left|t^{x x}\right|=\left|t^{x z}\right|=0$ and $\left|t^{z z}\right|=t$. Here $t=V_{d p \sigma}^{2} / \Delta \epsilon_{d p}$ is determined by the Mn- $d$ O- $p$ hybridisation $V_{d p \sigma}$ and the corresponding level splitting $\Delta \epsilon_{d p}$. All matrix elements are negative except $t^{x z}$ along x-direction, which is positive. The level splitting $\Delta E_{\mathbf{i}}=E_{\mathbf{i}}^{z}-E_{\mathbf{i}}^{x}$ can be controlled by uniaxial pressure or in the case of layered compounds even with hydrostatic pressure [28]. We shall focus here on the orbital degenerate case, i.e. $\Delta E_{\mathbf{i}}=0$, however we will keep the orbital energy term for the derivation of the orbital model.

The large local electron-electron repulsion $U$ is responsible for the insulating behavior for integer band-filling

$$
\begin{aligned}
H_{i n t}= & \sum_{\mathbf{i} a} U_{a} n_{\mathbf{i} a \uparrow} n_{\mathbf{i} a \downarrow}+U_{a b} \sum_{\mathbf{i}} n_{\mathbf{i} a} n_{\mathbf{i} b} \\
& +J_{a b} \sum_{\mathbf{i} \sigma \sigma^{\prime}} \tilde{d}_{\mathbf{i} a \sigma}^{\dagger} \tilde{d}_{\mathbf{i} b \sigma^{\prime}}^{\dagger} \tilde{d}_{\mathbf{i} a \sigma^{\prime}} \tilde{d}_{\mathbf{i} b \sigma} .
\end{aligned}
$$

Here $U_{a}, U_{a b}$ and $J_{a b}$ are the intra- and inter-orbital Hubbard and exchange interactions, respectively. The relevant valences of $\mathrm{Mn}$ are $\mathrm{Mn}^{3+}(\mathrm{S}=2)$ and $\mathrm{Mn}^{4+}(\mathrm{S}=3 / 2)$ as was pointed out already by Zener [13]. The Hamiltonian $H_{\text {band }}+H_{\text {int }}$ was considered by Zaanen and Oleś who showed that in general rather complex effective Hamiltonians result for transition metal ions with partially filled $d$ shell near orbital degeneracy [29]. Here a simplified approach is preferred, with the $t_{2 g}$ electrons of Mn ions forming core spins of $S=3 / 2$, and thus we restrict the electron interactions in $H_{i n t}$ to the $e_{g}$ bands. 
Even in the case with local orbital degeneracy there is one lower Hubbard band which is partially filled in the case of hole doping. With one $e_{g}$-electron per site these systems are Mott-insulators. Although the Jahn-Teller splitting can lead to a gap in the absence of $U$, it is not the primary reason for the insulating behavior for integer filling 30.

The interaction of itinerant electrons with the $(\mathrm{S}=3 / 2)$ core spins is given by

$$
H_{K o n d o}=-K \sum_{\mathbf{i} a \sigma \sigma^{\prime}} \vec{S}_{\mathbf{i}} \cdot d_{\mathbf{i} a \sigma}^{\dagger} \vec{\sigma}_{\sigma \sigma^{\prime}} d_{\mathbf{i} a \sigma^{\prime}}
$$

leading to parallel alignment of the d-electron spin with the spin $\vec{S}_{\mathbf{i}}$. Since the d-electron kinetic energy is favored by a parallel orientation of neighboring spins the ground state becomes ferromagnetic [13].

In the low-temperature ferromagnetic phase we may introduce an effective Hamiltonian which contains only the orbital degrees of freedom assuming a fully spinpolarized ferromagnet. The spin degrees of freedom can also be eliminated by high magnetic fields. This opens the possibility to investigate the orbital order independent of the spin degrees of freedom, and to shed light on the nontrivial question how the orbital order is changed upon doping with $e_{g}$-electrons or holes as well as the possible appearance of an orbital liquid state.

The resulting model has similarities to the $t$ - $J$ model, where the spin-indices $\sigma$ and $\sigma^{\prime}$ are now orbital indices. To stress the difference we use for the orbital indices the letters $a, b$ and $\alpha, \beta$. Unitary transformation $H_{\text {orb }}=e^{-S} H e^{S}$ 31 and the restriction to states without double occupancy leads to the orbital $t$ - $J$ model which has the following structure:

$$
H_{o r b}=\sum_{\mathbf{i} a} E_{a}^{\mathbf{i}} \tilde{d}_{\mathbf{i} a}^{\dagger} \tilde{d}_{\mathbf{i} a}+\sum_{\langle\mathbf{i} \mathbf{j}\rangle a b}\left(t_{\mathbf{i j}}^{a b} \tilde{d}_{\mathbf{i} a}^{\dagger} \tilde{d}_{\mathbf{j} b}+H . c .\right)+H_{o r b}^{\prime} .
$$

with the constraint that each site can be occupied by at most one electron, i.e. $\tilde{d}_{\mathbf{i} a}^{\dagger}=d_{\mathbf{i} a}^{\dagger}\left(1-n_{\mathbf{i} \bar{a}}\right)$. Here and in the following the index $\bar{a}$ denotes the orthogonal $e_{g}$ orbital with respect to orbital $a$. For one electron per site this model describes a Mott insulator. The orbital interaction $H_{\text {orb }}^{\prime}$ follows as a consequence of the elimation of doubly occupied sites with energy $U \sim U_{a b}-J_{a b}$.

$$
\begin{aligned}
H_{o r b}^{\prime}= & -\frac{1}{2} \sum_{\mathbf{j u \mathbf { u } ^ { \prime }}} \sum_{a b \alpha \beta} t_{\mathbf{j}+\mathbf{u} \mathbf{j}}^{\alpha \beta} t_{\mathbf{j} \mathbf{j}+\mathbf{u}^{\prime}}^{b a}\left(\frac{1}{U+E_{\mathbf{j}}^{\beta}-E_{\mathbf{j}+\mathbf{u}}^{\alpha}}\right. \\
& \left.+\frac{1}{U+E_{\mathbf{j}}^{b}-E_{\mathbf{j}+\mathbf{u}^{\prime}}^{a}}\right)\left[\delta_{\beta, b} \tilde{d}_{\mathbf{j}+\mathbf{u} \alpha}^{\dagger} \tilde{d}_{\mathbf{j} \bar{\beta}}^{\dagger} \tilde{d}_{\mathbf{j} \bar{b}^{b} \tilde{d}_{\mathbf{j}+\mathbf{u}^{\prime} a}}\right. \\
& \left.-\delta_{\beta, \bar{b}} \tilde{d}_{\mathbf{j}+\mathbf{u} \alpha}^{\dagger} \tilde{d}_{\mathbf{j} b}^{\dagger} \tilde{d}_{\mathbf{j} \beta} \tilde{d}_{\mathbf{j}+\mathbf{u}^{\prime} a}\right]
\end{aligned}
$$

Here $\mathbf{u}, \mathbf{u}^{\prime}=( \pm a, 0)$ or $(0, \pm a)$ are lattice unit vectors. The orbital interaction $H_{\text {orb }}^{\prime}=H_{\text {orb }}^{\prime(2)}+H_{\text {orb }}^{\prime(3)}$ consists of two types of contributions: (i) 2-site terms $\left(\mathbf{u}=\mathbf{u}^{\prime}\right)$, i.e. similar to the Heisenberg interaction in the standard $t$ $J$ model, yet more complex because of the nonvanishing off-diagonal $t^{a b}$, and (ii) 3-site hopping terms $\left(\mathbf{u} \neq \mathbf{u}^{\prime}\right)$ between second nearest neighbors. In the half-filled case, i.e. one $e_{g}$-electron per site, only the 2 -site interaction is operative and may induce some orbital order. In the presence of hole doping, i.e. for less than one $e_{g}$-electron per site, both the kinetic energy and the 3-site contributions in $H_{\text {orb }}^{\prime}$ lead to propagation of the holes and to a frustration of the orbital order.

The complexity of the model can be seen if we express the orbital interaction $H_{\text {orb }}^{\prime}$ in terms of pseudospin operators $T_{\mathbf{i}}^{z}=\frac{1}{2}\left(n_{\mathbf{i} a}-n_{\mathbf{i} b}\right), T_{\mathbf{i}}^{+}=\tilde{d}_{\mathbf{i} a}^{\dagger} \tilde{d}_{\mathbf{i} b}$ and $T_{\mathbf{i}}^{-}=\tilde{d}_{\mathbf{i} b}^{\dagger} \tilde{d}_{\mathbf{i} a}$. where $a$ and $b$ denote an orthogonal orbital basis in the $e_{g}$-space. To be specific we shall assume here $a(b)=z(x)$. Keeping only the two-site contributions, i.e. $\mathbf{u}=\mathbf{u}^{\prime}$, one obtains an anisotropic Heisenberg Hamiltonian for the orbital interactions:

$$
\begin{aligned}
H_{o r b}^{\prime(2)}=-\frac{2}{U} \sum_{\langle\mathbf{i}\rangle} & {\left[\left(t_{\mathbf{i j}}^{a a 2}+t_{\mathbf{i j}}^{b b^{2}}\right)\left(\frac{1}{4} n_{\mathbf{i}} n_{\mathbf{j}}-T_{\mathbf{i}}^{z} T_{\mathbf{j}}^{z}\right)\right.} \\
& -t_{\mathbf{i j}}^{a a} t_{\mathbf{i j}}^{b b}\left(T_{\mathbf{i}}^{+} T_{\mathbf{j}}^{-}+T_{\mathbf{i}}^{-} T_{\mathbf{j}}^{+}\right) \\
& +\left(t_{\mathbf{i j}}^{a b^{2}}+t_{\mathbf{i j}}^{b a^{2}}\right)\left(\frac{1}{4} n_{\mathbf{i}} n_{\mathbf{j}}+T_{\mathbf{i}}^{z} T_{\mathbf{j}}^{z}\right) \\
& -t_{\mathbf{i j}}^{a b} t_{\mathbf{i} \mathbf{j}}^{b a}\left(T_{\mathbf{i}}^{+} T_{\mathbf{j}}^{+}+T_{\mathbf{i}}^{-} T_{\mathbf{j}}^{-}\right) \\
& -\left(t_{\mathbf{i} \mathbf{j}}^{a a} t_{\mathbf{i j}}^{a b}-t_{\mathbf{i j}}^{b b} t_{\mathbf{i} \mathbf{j}}^{b a}\right)\left(T_{\mathbf{i}}^{z} T_{\mathbf{j}}^{+}+T_{\mathbf{i}}^{z} T_{\mathbf{j}}^{-}\right) \\
& \left.-\left(t_{\mathbf{i j}}^{a a} t_{\mathbf{i} \mathbf{j}}^{b a}-t_{\mathbf{i} \mathbf{j}}^{b b} t_{\mathbf{i} \mathbf{j}}^{a b}\right)\left(T_{\mathbf{i}}^{+} T_{\mathbf{j}}^{z}+T_{\mathbf{i}}^{-} T_{\mathbf{j}}^{z}\right)\right] .
\end{aligned}
$$

The orbital interaction $H^{\prime(2)}$ is equivalent to that studied by Ishihara et al. 221,28. Since we are interested here in the effect of holes on the orbital order we will generally base our study on $H_{\text {orb }}^{\prime}(6)$ which includes the 3 -site hopping processes as well.

We note that in the special case $t_{\mathbf{i} \mathbf{j}}^{a b}=\delta_{a b} t$ and $E_{\mathbf{i}}^{a}=0$ equations (5) and (6) are identical to the usual $t$ - $J$ model ( with $J=4 t^{2} / U$ and including 3 -site processes). For the orbital model we shall adopt the same convention for the orbital exchange coupling, i.e. $J=4 t^{2} / U$.

The orbital degrees of freedom in combination with strong correlations (i.e. no doubly occupied sites are allowed) is expected to lead to incoherent motion of holes, although quasiparticle formation with reduced spectral weight is a plausible expectation on the basis what is known about the usual $t$ - $J$ model.

In this paper we focus on the orbital degenerate case with $E_{i}^{a}=E_{i}^{b}=0$. A detailed study of the influence of a finite level splitting will be presented elsewhere.

\section{OPTICAL CONDUCTIVITY}

The frequency dependent conductivity consists of two parts [32] 


$$
\sigma_{0}(\omega)=\sigma(\omega)+2 \pi e^{2} D_{c} \delta(\omega) .
$$

The $\delta$-function contribution is proportional to the charge stiffness $D_{c}$, which vanishes in insulators. This contribution is broadened into a usual Drude peak in the presence of other scattering processes like impurities which are not contained in the present model. The finite frequency absorption (or regular part) $\sigma(\omega)$ is determined by the current-current correlation function:

$$
\sigma(\omega)=\frac{1-e^{-\omega / T}}{N \omega} \operatorname{Re} \int_{0}^{\infty} d t e^{i \omega t}\left\langle j_{x}(t) j_{x}\right\rangle .
$$

For the derivation of the current operator we introduce twisted boundary conditions via Peierls construction

$$
t_{\mathbf{j}+\mathbf{u} \mathbf{j}}^{a b}(\vec{A})=t_{\mathbf{j}+\mathbf{u} \mathbf{j}}^{a b} \exp \left(-i \frac{e}{\hbar} \int_{\mathbf{j}}^{\mathbf{j}+\mathbf{u}} \vec{A}(x) \overrightarrow{d x}\right) .
$$

Assuming $\vec{A}=\left(A_{x}, A_{y}, 0\right)$ we obtain from the kinetic energy operator the x-component of the current operator $j_{x}=\partial H\left(A_{x}\right) / \partial A_{x}$ :

$$
j_{x}^{(1)}=-i e \sum_{\mathbf{j}+\mathbf{u} a b} t_{\mathbf{j}+\mathbf{u} \mathbf{j}}^{a b} u_{x} \tilde{d}_{\mathbf{j}+\mathbf{u} a}^{\dagger} \tilde{d}_{\mathbf{j} b}
$$

An additional contribution follows from the 3-site term in Eq.(6)

$$
\begin{aligned}
j_{x}^{(3)} & =\frac{i e}{2 U} \sum_{\mathbf{j} \mathbf{u} \mathbf{u}^{\prime}} \sum_{a b \alpha \beta} t_{\mathbf{j}+\mathbf{u} \mathbf{j}}^{\alpha \beta} t_{\mathbf{j} \mathbf{j}+\mathbf{u}^{\prime}}^{b a}\left(u_{x}-u_{x}^{\prime}\right) O_{b a}^{\alpha \beta}\left(\mathbf{j}, \mathbf{u}, \mathbf{u}^{\prime}\right), \\
O_{b a}^{\alpha \beta} & =\delta_{\beta, b} \tilde{d}_{\mathbf{j}+\mathbf{u} \alpha}^{\dagger} \tilde{d}_{\mathbf{j} \bar{\beta}}^{\dagger} \tilde{d}_{\mathbf{j} b} \tilde{d}_{\mathbf{j}+\mathbf{u}^{\prime} a}-\delta_{\beta, \bar{b}} \tilde{d}_{\mathbf{j}+\mathbf{u} \alpha}^{\dagger} \tilde{d}_{\mathbf{j} b}^{\dagger} \tilde{d}_{\mathbf{j} \beta} \tilde{d}_{\mathbf{j}+\mathbf{u}^{\prime} a},
\end{aligned}
$$

where the expression $O_{b a}^{\alpha \beta}\left(\mathbf{j}, \mathbf{u}, \mathbf{u}^{\prime}\right)$ is an abbreviation for the operator within the last brackets of Eq.(6).

We want to stress here that the orbital order in the undoped case is determined exclusively by the (two-site) orbital interaction (7), while the 3 -site processes in (6) only contribute in the doped case. Nevertheless the motion of holes and transport in general is influenced in a significant way by the 3 -site current operator $j_{x}^{(3)}$. In fact from studies of the $t-J$ model it is known that $\sigma(\omega)$ is not only quantitatively but even qualitatively changed by these terms 18,19 . Although the 3 -site terms are usually ignored in studies of the $t-J$ model they are an important part of the strong coupling model and should not be droped in studies of the conductivity. One of the aims in our study of the orbital model is to analyse the effect of these 3 -site terms in the model.

\section{A. Finite Temperature Lanczos Method (FTLM)}

For the calculation of $\sigma(\omega)$ we use a generalization of the exact diagonalization technique for finite temperature developed by Jaklič and Prelovšek [23]. In this approach the trace of the thermodynamic expectation value is performed by a Monte-Carlo sampling. The current-current correlation function in (9):

$$
C(\omega)=\operatorname{Re} \int_{0}^{\infty} d t e^{i \omega t}\left\langle j_{x}(t) j_{x}\right\rangle .
$$

can be rewritten by introducing a complete set of basis functions $|r\rangle$ for the trace, and eigenfunctions $\left|\Psi_{j}^{r}\right\rangle$ and $\left|\tilde{\Psi}_{j}^{r}\right\rangle$ of $H$ with eigenvalues $E_{i}^{r}$ and $\tilde{E}_{j}^{r}$ (here $r$ is an irrelevant label, which will get its meaning below):

$$
\begin{array}{r}
C(\omega) \approx \frac{\pi}{Z} \sum_{r=1}^{R} \sum_{i, j=1}^{M} e^{-\beta E_{i}^{r}\left\langle r \mid \Psi_{i}^{r}\right\rangle\left\langle\Psi_{i}^{r}\left|j_{x}\right| \tilde{\Psi}_{j}^{r}\right\rangle} \\
\left\langle\tilde{\Psi}_{j}^{r}\left|j_{x}\right| r\right\rangle \delta\left(\omega+E_{i}^{r}-\tilde{E}_{j}^{r}\right)
\end{array}
$$

with

$$
Z \approx \sum_{r=1}^{R} \sum_{i=1}^{M} e^{-\beta E_{i}^{r}}\left|\left\langle r \mid \Psi_{i}^{r}\right\rangle\right|^{2} .
$$

These relation are exact if one uses complete sets. The FTLM is based on two approximations when evaluating Eqs.(14) for $C(\omega)$ and (15) for the partition function $Z$ : (1) The trace is performed over a restricted number $R$ of random states $|r\rangle$, and (2) the required set of eigenfunctions of $H$ is generated by the Lanczos algorithm starting from the initial states $\left|\Phi_{0}^{r}\right\rangle=|r\rangle$ and $\left|\tilde{\Phi}_{0}^{r}\right\rangle=j_{x}|r\rangle / \sqrt{\left\langle r\left|j_{x}^{2}\right| r\right\rangle}$, respectively. This procedure is truncated after $M$ steps and yields the relevant intermediate states for the evaluation of $C(\omega)$.

Detailed tests [23] have shown that the results get very accurate already if $R, M \ll N_{s t}$, where $N_{s t}$ is the dimension of the Hilbert space. Therefore one is saving a lot of computational effort because only matrices of dimension $M \times M$ have to be diagonalized, i.e. much smaller than the dimension $N_{s t} \times N_{s t}$ of the full Hamiltonian $H$.

Since in our problem $T_{\text {tot }}^{z}$ does not commute with the Hamiltonian $H_{\text {orb }}$ (5) the calculations for the orbital model are restricted to smaller clusters than in the $t-J$ case, where different $S_{\text {tot }}^{z}$ subspaces can be treated separately.

\section{B. Results}

Typical results for the frequency and temperature dependence of $\sigma(\omega)$ for a two-dimensional 10-site cluster with 2 holes, i.e. corresponding to a doping concentration $x=0.2$, are shown in Fig. 1 with and Fig. 2 without 3 -site hopping terms, respectively. As characteristic feature of the broad continuum we note: (a) the increase of the absorption $\sigma(\omega)$ with decreasing temperature, and (b) the width of the distribution $\omega_{1 / 2} \sim 2.5 t$ measured at half-maximum. This half-width $\omega_{1 / 2}$ is independent of 
temperature. We further note that similar calculations for a $3 \mathrm{D}$ cluster yield a slightly larger value $\omega_{1 / 2} \sim 3.5 t$.

If we compare this latter $(3 \mathrm{D})$ value with $\omega_{1 / 2}^{\exp } \sim 0.7$ $\mathrm{eV}$ found from the data of Okimoto et al. (for $x=0.175$ and $T=9 \mathrm{~K}$ ), we obtain an experimental estimate for the parameter $t: t^{o p t} \sim 0.2 \mathrm{eV}$. For comparison a rough theoretical estimate based on Harrison's solid state table would yields $t=V_{d p \sigma}^{2} / \Delta \epsilon \sim 0.4 \mathrm{eV}$.

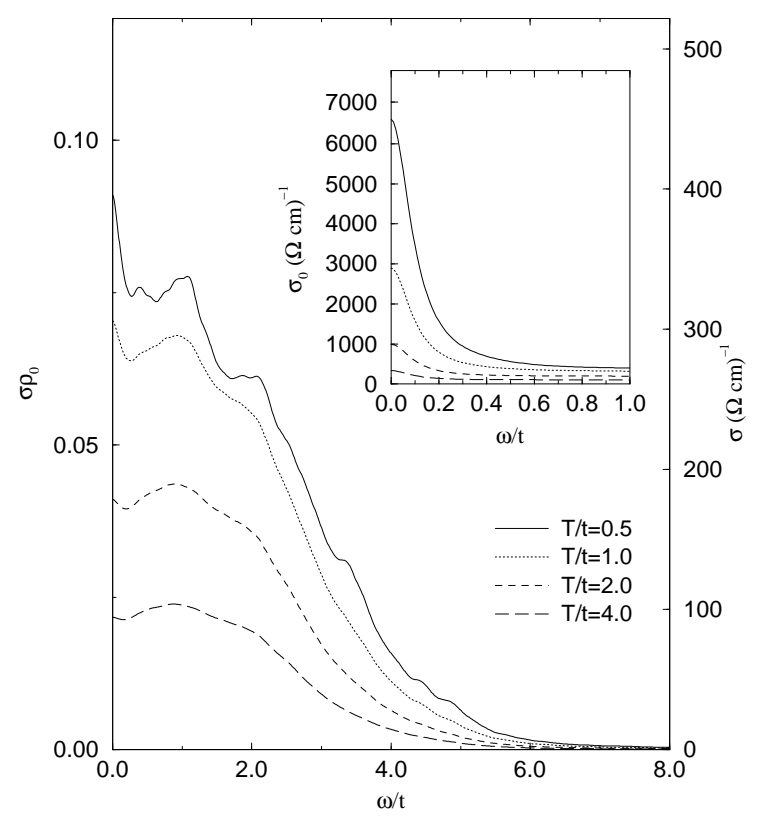

FIG. 1. Frequency dependent regular part of the optical conductivity $\sigma(\omega)$ for a $\mathrm{N}=10$ site plane with $N_{e}=8$ electrons and $\mathrm{J}=0.25 \mathrm{t}(\mathrm{U}=16 \mathrm{t})$ at different temperatures. The calculation includes the 3 -site terms. The inset shows the optical conductivity $\sigma_{0}(\omega)$ including the Drude-part. The spectra were broadened with $\Gamma=0.1 \mathrm{t}$.

Although $\sigma(\omega)$ shows the anomalous increase with decreasing temperature, we do not claim here that the orbital model accounts for the full temperature dependence in the ferromagnetic phase $\left(T<T_{c}\right)$. The description of the complete $T$-dependence certainly requires the analysis of the degenerate-orbital KLM, i.e. the additional inclusion of the spin degrees of freedom. Yet we believe that the results can be compared with the data by Okimoto et al. in the low- $T$ limit, i.e. in the regime where the magnetization is saturated.

To allow comparison with experimental data we present the conductivity in dimensionless form and with the proper dimensions of a 3D conductivity, assuming that the $3 \mathrm{D}$ system is formed by noninteracting layers. For a cubic lattice $\rho_{0}=\hbar a / e^{2}$. We further note that calculations for a small $3 \mathrm{D}$ cluster yield a rather similar $\sigma(\omega)$ distribution. If we consider $\mathrm{La}_{1-x} \mathrm{Sr}_{x} \mathrm{MnO}_{3}$ with lattice constant $a=5.5 \AA$ and $\hbar / e^{2}=4.11 \mathrm{~K} \Omega$ we ob- tain $\rho_{0}=0.23 \cdot 10^{-3} \Omega \mathrm{cm}$. Hence the low frequency limit of the regular part of the conductivity $\sigma(\omega \rightarrow 0)=$ $\sigma_{0}^{i n c} \sim 0.3-0.4 \cdot 10^{3}(\Omega \mathrm{cm})^{-1}$. This is consistent with the order of magnitude for the low frequency limit of the incoherent part of the $\sigma(\omega)$ data of Okimoto et al. for $\mathrm{La}_{1-x} \mathrm{Sr}_{x} \mathrm{MnO}_{3} \sigma_{0}^{i n c} \sim 0.4 \cdot 10^{3}\left(0.3 \cdot 10^{3}\right)(\Omega \mathrm{cm})^{-1}$ for the doping concentrations $x=0.175(0.3)$, respectively, at $T=10 \mathrm{~K}$.

Hence we conclude that besides the energy scale also the absolute value of the incoherent $\sigma(\omega)$ spectrum is consistent with the orbital model. We stress that the value (order of magnitude) of $\sigma_{0}^{i n c}$ is essentially fixed by the conductivity sum rule and the scale $\omega_{1 / 2}$, as long as the conductivity is predominantly incoherent.

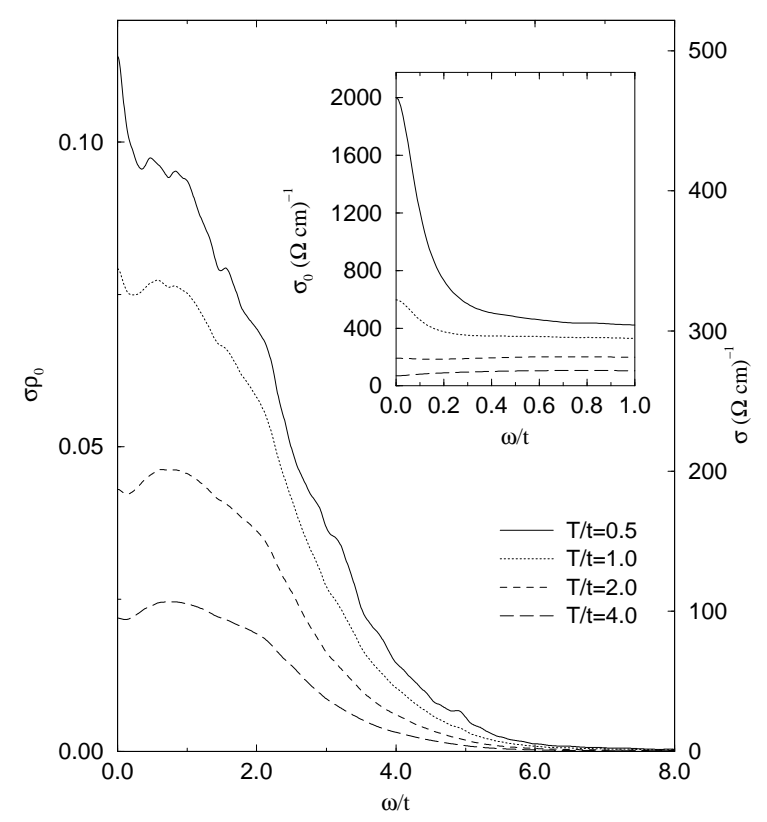

FIG. 2. Optical conductivity as in Fig. 1 but without 3 -site contributions. Due to the stronger incoherence there is a smaller Drude peak (inset) and a smaller DC-conductivity.

The insets in Fig.1 and show $\sigma_{0}(\omega)$ (8) with the Drude absorption at low frequency included, where we use an ad hoc chosen smearing parameter $\Gamma=0.1 t$. This value corresponds to the experimental width $\Gamma \sim 0.02$ eV taken from the (small) Drude peak observed by Okimoto et al. [4]. While our model yields the weight of the Drude peak $D_{c}$, it does not give $\Gamma$, which is due to extrinsic processes (e.g. scattering from impurities and grain boundaries). The charge stiffness $D_{c}$ together with $\Gamma$ determines the DC-conductivity.

Adopting the experimental estimate for $\Gamma$ we find for the example in Fig.1 (inset) for the low temperature DCconductivity $\sigma_{D C} \sim 6.5 \cdot 10^{3}(\Omega \mathrm{cm})^{-1}$ and for the resistivity $\rho \sim 0.15 \cdot 10^{-3} \Omega \mathrm{cm}$, respectively. For comparison, the experimental range of resistivities is e.g. 
$0.1-1.0 \cdot 10^{-3} \Omega \mathrm{cm}$ in the ferromagnetic metallic phase of $\mathrm{La}_{1-x} \mathrm{Sr}_{x} \mathrm{MnO}_{3}$ 33. As we shall see below, the Drude weight and therefore the DC-conductivity depend considerably on the value of the exchange coupling $J$, and whether the 3-site hopping processes in the model are taken into account or not. These terms have a strong effect on the coherent motion of charge carriers.

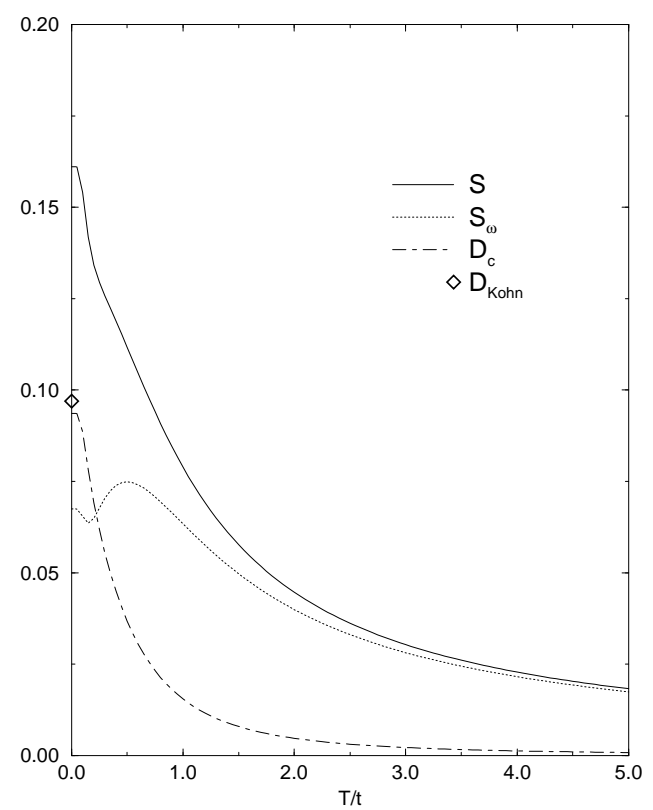

FIG. 3. Temperature dependence of the kinetic energy S (solid line), the incoherent spectral weight $S_{\omega}$ (dotted) and the Drude weights $D_{c}$ (dash-dotted line) and $D_{K o h n}$ (diamond) for a $\mathrm{N}=10$ site plane with $N_{e}=8$ electrons and $\mathrm{J}=0.25 \mathrm{t}$ $(\mathrm{U}=16 \mathrm{t})$ in units of $t / \rho_{0} e^{2}$.

The charge stiffness $D_{c}$ can be determined in two ways: (a) using Kohn's relation [34, or (b) via the optical sum rule which relates the integrated spectral weight of the real part of the conductivity to the average kinetic energy:

$$
\int_{-\infty}^{\infty} \sigma_{0}(\omega) d \omega=-\frac{\pi e^{2}}{N}\left\langle H_{x x}^{k i n}\right\rangle .
$$

Here $H^{\text {kin }}$ contains two contributions: (a) the usual kinetic energy $\sim t(5)$ and (b) the 3 -site hopping processes $\sim t^{2} / U$ in Eq. (6). The case with off-diagonal hopping considered here is a generalization of the sum rules for the $t$ - $J$ model [35] and for the $t$ - $J$ model including 3 -site terms 18,20]. Together with (8) this implies

$$
D_{c}=-\frac{1}{2 N}\left\langle H_{x x}^{k i n}\right\rangle-\frac{1}{\pi e^{2}} \int_{0^{+}}^{\infty} \sigma(\omega) d \omega .
$$

In the following we shall use $D_{c}=S-S_{\omega}$ as abbreviation for this equation, where $S$ denotes the sum rule expression and $S_{\omega}$ the finite frequency absorption. Figure 3 shows the temperature variation of the sum rule (kinetic energy), the finite frequency absorption $S_{\omega}$ and the Drude weight $D_{c}$ for the model including the 3 -site terms.

Whereas at high temperature the sum rule is essentially exhausted by the finite frequency absorption $\sigma(\omega)$, we find at low temperatures a significant increase of the Drude weight. In this particular case $D_{c}$ contributes about $40 \%$ to the sum rule at $T / t=0.3$.

The conductivity data shown in Figs. 1 and 2 is characteristic for a 2D orbital liquid state, which is stabilized by thermal fluctuations. The significant increase of coherency in Fig. 3 below $T / t=0.3$ is due to $x^{2}-y^{2}$ orbital ordering in the planar model (see discussion in Section IV and in particular Fig. 10). This is characteristic for the $2 \mathrm{D}$ version of the orbital $t$ - $J$ model and does probably not occur in cubic systems for which an orbital liquid ground state was proposed [10]. Therefore we consider our $T / t=0.3$ data for $D_{c}$ to be more appropriate for a comparison with the low-temperature $3 \mathrm{D}$ data than the $T=0$ values, which are enhanced due to orbital order.

We have also calculated $D_{c}$ for $T=0$ using Kohn's relation 34

$$
D_{K o h n}=\left.\frac{1}{2 N} \frac{\partial^{2} E_{0}(\Phi)}{\partial \Phi^{2}}\right|_{\Phi=\Phi_{0}},
$$

which yields a consistent value (Fig. 3). Here $\Phi=e a A_{\alpha} / \hbar$ is the Peierls phase induced by the applied vector potential with component $A_{\alpha}$. For the evaluation of Eq.18 we follow Ref. 18 and first search for the minimum of the ground state energy $E_{0}$ with respect to the vector potential 36], and then calculate the curvature. Since this procedure is quite cumbersome, we have determined most of our $D_{c}$ data via the sum rule. The resulting sets of data show systematic trends as function of $J$ and doping.

From these results we expect in analogy with the $t-J$ model that the single-particle electron Green's function is characterized by a pronounced quasiparticle peak to explain such a large fraction of coherent transport.

From Fig. 3 we also see that the weight under $\sigma(\omega)$, i.e. $S_{\omega}$, does not further increase for temperatures below $T=0.5 t$, although the temperature variation in Fig.1 seems to suggest a significant further increase towards lower temperatures. Conductivity data for $T<0.5 t$ is not shown in Figs. 1 and 2 because it shows pronounced discrete level structure, and probably requires larger clusters for a careful study. Nevertheless it appears that $\sigma(\omega)$ develops a pseudogap in the orbital ordered phase [37. Integrated quantities on the other hand are much less influenced by such effects. In view of the significant variation of the $\sigma(\omega)$ continuum in the experimental data [4] in the range $0<T<T_{c}$, this suggests: (a) that the spectral change below $T_{c}$ is a combined effect of orbital- 
and spin-degrees of freedom and hence requires the full Kondo lattice model (1) for its description, and (b) only the experimental data for $T \rightarrow 0$ can be compared directly with the orbital model.

In the following we wish to shed more light on the role of the 3 -site processes in the orbital Hamiltonian and in the current operator. The importance of the 3-site term becomes particularly clear from the $J$-dependence of the sum rule $S$ (Fig. 4 ) and the charge stiffness $D_{c}$ (Fig.5) taken at temperature $T / t=0.3$. These low temperature values of $S$ and $D_{c}$ are approximatively independent of the exchange parameter $J$ in the absence of 3 -site terms. A similar observation was made earlier for the $t-J$ model 38, 18. Moreover the sum rule $S$ is proportional to the doping concentration $x$ for the 3 cases shown $(x=0.125$, 0.2 , and 0.3 ). When 3 -site terms are taken into account both $S$ and $D_{c}$ acquire a component which increases linearly with $J$. These general features are fully consistent with results for the generalized $t-J$ model 18 . In particular we see that the 3-site hopping terms introduce more coherence, for example for $J=0.5 D_{c} \sim 0.012$ without and 0.08 with 3 -site terms.

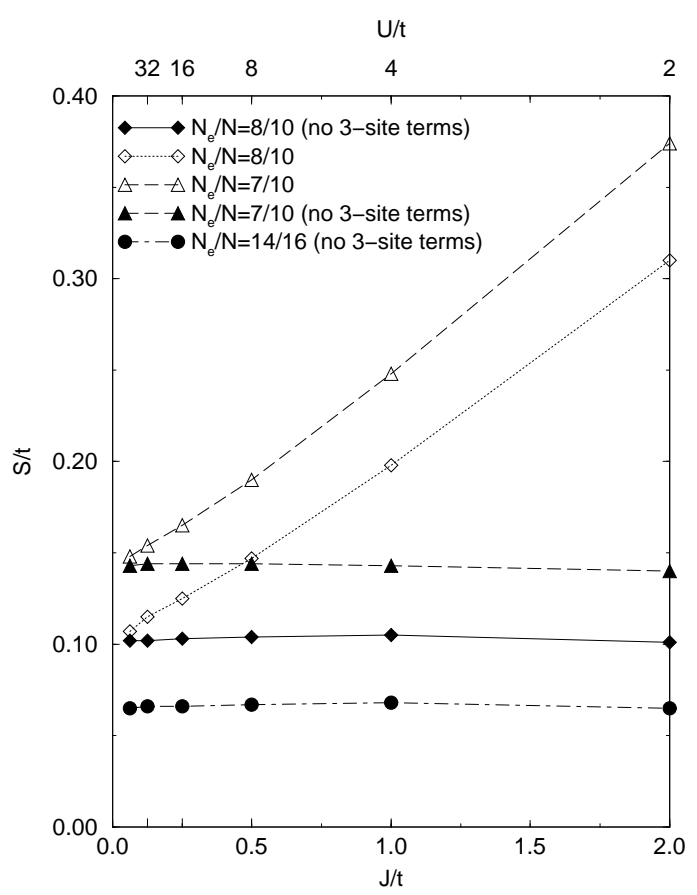

FIG. 4. Kinetic energy $\mathrm{S}$ versus $\mathrm{J}$ for a $\mathrm{N}=10$ site $2 \mathrm{D}$ cluster with $N_{e}=7$ and 8 electrons, with (full) and without (open symbols) the 3 -site terms $(T / t=0.3)$, respectively. The dash-dotted line (circles) represents a 16 site cluster with $N_{e}=14$ and without 3-site terms.

The change due to the 3 -site terms is particularly large for the charge stiffness, which defines the weight of the low frequency Drude peak. The relative weight in the Drude peak $D_{c} / S$ is shown in Fig. 6 as function of $J=$
$4 t^{2} / U$. Small changes in $J$ lead to considerable changes in the Drude weight and the coherent motion of carriers. For $J=0.25(0.5)$ the 3 -site terms lead to an increase of $D_{c}$ by a factor of $3(5)$, respectively, for the 8 electron case.

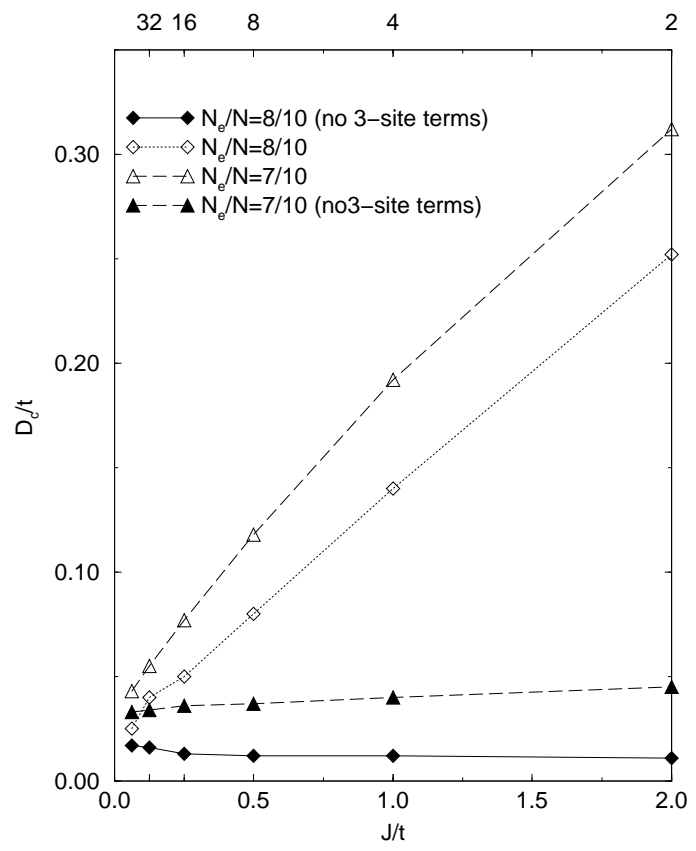

FIG. 5. Drude weight $D_{c}$ versus $J$ calculated via the sum-rule (17) for a $\mathrm{N}=10$ site planar cluster with $N_{e}=7$ and 8 $e_{g}$ electrons, with and without the 3 -site terms, respectively.

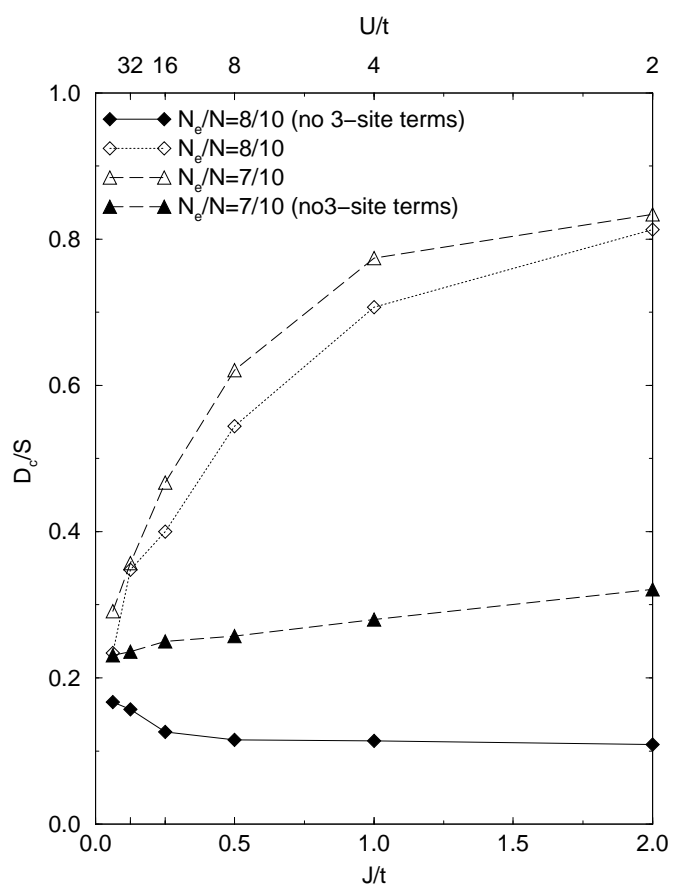


FIG. 6. The relative weight of the Drude peak $D_{c} / S$ vs. $J$ with and without 3 -site terms for a 10-site cluster with 2 and 3 holes $(\mathrm{x}=0.2$ and 0.3$)$, respectively, at temperature $T / t=0.3$.

When we take the value $t \sim 0.2 \mathrm{eV}$, which we determined from the comparison of $\sigma(\omega)$ with the experimental data, and $U \sim 3 \mathrm{eV}$ [21], we obtain $U / t \sim 15$ and $J / t=4 t / U \sim 0.25(J=0.05 \mathrm{eV})$. For such a value for $J$ we expect a relative Drude weight $D_{c} / S \sim 0.4$, which is larger than the experimental ratio $D_{c} / N_{\text {eff }} \sim 0.2$ found by Okimoto et al. for $x=0.175$, identifying here the effective number of carriers $N_{\text {eff }}$ [ [4] with $S$. The small value for $D_{c} / N_{\text {eff }}$ found in the experiments suggests that $J$ is not larger than the value estimated, otherwise we would expect a much too large relative Drude weight. A larger value for $U$ would also have the effect to reduce $D_{c}$. However, we have to stress here that certainly calculations on larger clusters must be performed, before possible finite size effects in $D_{c}$ can be quantified.

\section{Comparison with other work}

The optical conductivity for the orbital non-degenerate Kondo latice model was studied by Furukawa using the dynamical mean-field approximation [9]. An important result of this calculation is, that the weight of intraband excitations within the lower exchange-split band should be proportional to the normalized ferromagnetic magnetization $M / M_{\text {sat }}$. Experimentally, however, $N_{\text {eff }}(\sim S)$ is still increasing when temperature is lowered even though $M$ is already saturated. This contradiction to the prediction of the simple double-exchange model implies that some other large-energy-scale scattering mechanism survives at low temperature, where the spins are frozen [4]. The dynamical mean field theory yields only one low energy scale, and not two, i.e. there is no Drude peak plus incoherent structure.

Recently Shiba et al. [11] analysed the noninteracting two-band model (2) (i.e. without constraint) and argued that the interband transitions within the $e_{g}$ orbitals may explain the anomalous absorption in the ferromagnetic phase of $\mathrm{La}_{1-x} \mathrm{Sr}_{x} \mathrm{MnO}_{3}$. While the frequency range of the interband transition is found consistent with the anomalous absorption, the structure of $\sigma(\omega)$ differs. In particular the noninteracting model leads to $\sigma(\omega) \sim \omega$ for small $\omega$. Moreover they calculated the ratio $S_{\omega} / D_{c} \sim 0.85$, i.e. they find more weight in the Drude peak than in the regular part of the conductivity $\sigma(\omega)$. The ratio is found to be rather insensitive to doping in the range $0.175<x<0.3$. This result differs considerably from the experimental data of Okimoto et $a l$ where this ratio is about 4 for $x=0.175$.

The nature of the orbital fluctuations in the ferromagnetic state and their effect on the optical conductivity was studied recently by Ishihara et al. 10 using slave fermions coupled to bosonic orbital excitations. They deal with the concentration regime $x t \gg J$, where the kinetic energy is expected to dominate the orbital exchange energy. Ishihara et al. arrive at the conclusion that the quasi two-dimensional nature of the orbital fluctuations leads to an orbital liquid in (3D) cubic systems. The orbital disorder is treated numerically in a static approximation. The $\sigma(\omega)$ spectrum obtained by this approach for $T=0.1 t$ has a quite similar shape as the experimental spectrum at low temperature (with $\omega_{1 / 2} \sim 3 t$ ), however, because of the assumed static disorder, there is no Drude component. A further problem with the orbital liquid, as mentioned by the authors, is the large entropy expected for the orbital disordered state which seems to be in conflict with specific heat measurements [39].

A microscopic theory of the optical conductivity based on a slave boson parametrization which combines strong correlations and orbital degeneracy was recently developed by Kilian and Khaliullin [40] for the ferromagnetic state at zero temperature. Their approach yields a highly incoherent spectrum due to the scattering of charge carriers from dynamical orbital fluctuations, and a Drude peak with strongly reduced weight. However $\sigma(\omega)$ is depressed at low frequency in this calculation for the orbital model and an additional electron-phonon mechanism is invoked to obtain results similar to the experimental spectral distribution. This theory further accounts for the small values of the specific heat and therefore supports the orbital liquid scenario for the cubic systems [10.

We note that the proposed orbital liquid state [10] is a property of the cubic systems, and not a property of the $2 \mathrm{D}$ or quasi-2D versions of the orbital model. In the planar model the cubic symmetry is broken from the very beginning and holes presumably cannot restore it. This is different from the physics of the $t$ - $J$ model, where holes restore the spin rotational symmetry (which is spontaneously broken in the antiferromagnetic ordered phase), because this symmetry is respected by the $t$ - $J$ Hamiltonian in any dimension.

To obtain a deeper understanding of our results for the conductivity, we shall analyse in the next Section the structure of the orbital correlations in the doped and undoped two-dimensional orbital model.

\section{ORBITAL CORRELATIONS AND DOPING DEPENDENCE}

In the absence of holes the anisotropic Heisenberg interaction (7) will lead to an orbital order below a certain temperature $\sim J$. Doping will destroy this order and lead either to a disordered orbital liquid, or may generate a new kind of ordered state which optimizes the kinetic energy of the holes. To investigate this question for an anisotropic model it is in general not sufficient 
to calculate e.g. the correlation function $<T_{\mathbf{i}}^{z} T_{\mathbf{j}}^{z}>$ defined with respect to the original orbital basis. In the following we introduce a local orthogonal transformation of the orbitals on different sublattices by angles $\phi$ and $\psi$, respectively. On the A-sublattice:

$$
\begin{aligned}
|\tilde{z}\rangle & =\cos (\phi)|z\rangle+\sin (\phi)|x\rangle \\
|\tilde{x}\rangle & =-\sin (\phi)|z\rangle+\cos (\phi)|x\rangle .
\end{aligned}
$$

This amounts to new operators, e.g.

$$
\begin{aligned}
\tilde{T}_{i}^{z} & =\cos (2 \phi) T_{i}^{z}+\sin (2 \phi) T_{i}^{x} \\
\tilde{T}_{i+R}^{z} & =\cos (2 \psi) T_{i+R}^{z}+\sin (2 \psi) T_{i+R}^{x}
\end{aligned}
$$

and new correlation functions, e.g. $\left\langle\tilde{T}_{\mathbf{i}}^{z} \tilde{T}_{\mathbf{j}}^{z}\right\rangle$ is defined as

$$
\begin{aligned}
\left\langle\tilde{T}_{i}^{z} \tilde{T}_{i+R}^{z}\right\rangle & =\cos (2 \phi) \cos (2 \psi)\left\langle T_{i}^{z} T_{i+R}^{z}\right\rangle \\
& +\sin (2 \phi) \sin (2 \psi)\left\langle T_{i}^{x} T_{i+R}^{x}\right\rangle \\
& +\cos (2 \phi) \sin (2 \psi)\left\langle T_{i}^{z} T_{i+R}^{x}\right\rangle \\
& +\sin (2 \phi) \cos (2 \psi)\left\langle T_{i}^{x} T_{i+R}^{z}\right\rangle
\end{aligned}
$$

The angles $\phi$ and $\psi$ are chosen such that the nearest neighbor correlation function $\left\langle\tilde{T}_{\mathbf{i}}^{z} \tilde{T}_{\mathbf{j}}^{z}\right\rangle$ takes a maximal (ferromagnetic) value. With this convention antiferromagnetic (AF) orbital order is accounted for by the choice of the local quantization axis, that is by the values of $\phi$ and $\psi$.

For the undoped 2D system (x-y plane) these angles are $\phi=45^{\circ}$ and $\psi=135^{\circ}$ corresponding to an $\frac{1}{\sqrt{2}}(|x\rangle+|z\rangle)$ and $\frac{1}{\sqrt{2}}(|x\rangle-|z\rangle)$ order at low temperatures (see Fig. 7). $\left\langle\tilde{T}_{i}^{z} \tilde{T}_{i+R}^{z}\right\rangle$ takes a value only slightly smaller than 0.25 at low temperature (Fig. 8), which implies that quantum fluctuations are small in this state. Hence the orbital correlation function $\left\langle\tilde{T}_{i}^{z} \tilde{T}_{i+R}^{z}\right\rangle$ of the undoped orbital model is alternating (i.e. antiferromagnetic in the pseudo-spin language for the orbital degrees of freedom). That the AF-order seems to be established at a finite temperature for the small clusters means that the correlation length $\xi$ gets larger than the systemsize L. At small temperatures the correlation functions have about the same value independent of $R$, i.e. the data shows no spatial decay. Above $T / t=0.1$ the orbital correlations are small and show a pronounced spatial decay, which can be considered as a signature of a thermally disordered orbital liquid state.

Experimentally the coexistence of antiferromagnetic (or staggered) orbital order and ferromagnetic spin order has been established in the low-doping regime of $\mathrm{LaMnO}_{3}$ 41,42. It should be recalled that $\mathrm{LaMnO}_{3}$ has an Atype antiferromagnetic spin structure [43], where ferromagnetic layers are coupled antiferromagnetically along the $c$-axis. In the orbital ordered case the $\mathrm{MnO}_{6}$ octahedra are deformed, and $\mathrm{Mn} 3 d_{3 x^{2}-r^{2}}$ and $3 d_{3 y^{2}-r^{2}}$ orbitals with orientation along the $x$ - and $y$-axis, respectively, have been considered as relevant occupied orbitals 444,45. For the planar model we find here the alternate occupation of $\frac{1}{\sqrt{2}}(|x\rangle-|z\rangle)$ and $\frac{1}{\sqrt{2}}(|x\rangle+|z\rangle)$ orbitals which are also oriented along the $x$ - and $y$-axis, however they differ with respect to their shape (and have pronounced lobes along $z$ ) 46. .

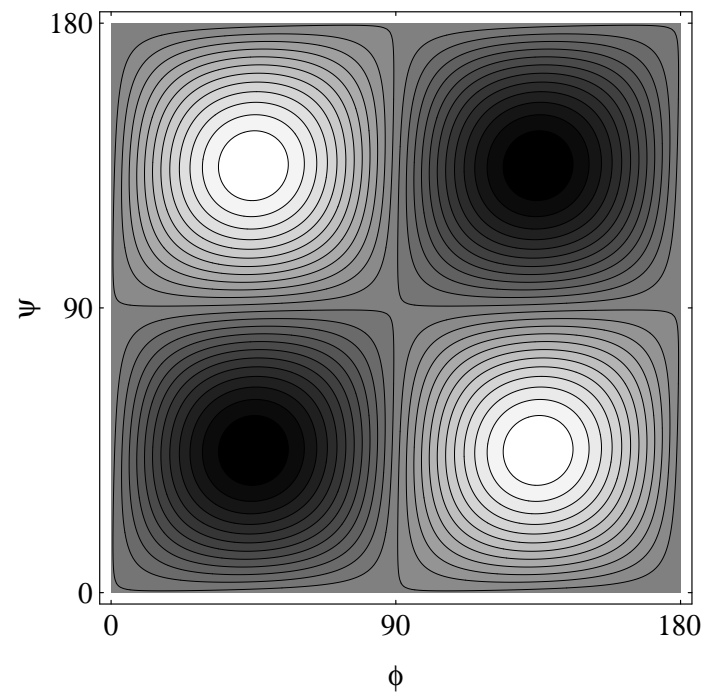

FIG. 7. Contourplot of the rotated nearest neighbor orbital correlation function $\left\langle\tilde{T}_{i}^{z} \tilde{T}_{i+R}^{z}\right\rangle, R=(1,0)$, as function of the angles $\phi$ and $\psi$ for a $\mathrm{N}=10$ site planar cluster with $N_{e}=10$ electrons, $\mathrm{J}=0.25 \mathrm{t}(\mathrm{U}=16 \mathrm{t})$ and $\mathrm{T}=0.01 \mathrm{t}$. White regions correspond to positive (ferromagnetic) and black areas to negative (antiferromagnetic) correlation functions, i.e. $\left\langle\tilde{T}_{i}^{z} \tilde{T}_{i+R}^{z}\right\rangle>0.23(<-0.23)$, respectively. The 25 contour lines are chosen equidistant in the intervall $[-1 / 4,+1 / 4]$.

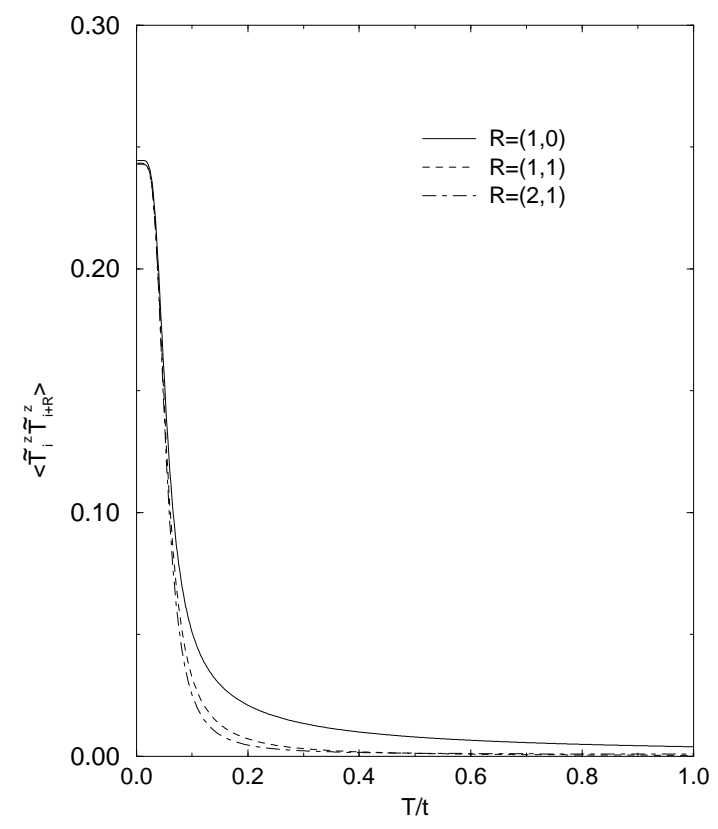


FIG. 8. Temperature dependence of $\left\langle\tilde{T}_{i}^{z} \tilde{T}_{i+R}^{z}\right\rangle$ with $\phi=45^{\circ}$ and $\psi=135^{\circ}$ for nearest-neighbors and $\psi=45^{\circ}$ for next-nearest neighbors, respectively. Results are shown for different distances $\mathrm{R}$ for an undoped $\mathrm{N}=10$ site planar cluster. Parameters as in Fig. 7. The strong increase for $T / t<0.1$ is due to the onset of alternating orbital order.

Two holes are sufficient to remove the antiferromagnetic orbital order and establish a ferromagnetic orbital order $\left(\phi=\psi=0^{\circ}\right)$ as can be seen from Figs. 9 and 10 . This corresponds to preferential occupation of orbitals with symmetry $x^{2}-y^{2}$. This can be considered as a kind of double exchange mechanism in the orbital sector. An interesting feature in Fig 10 for the doped case is the fact that the correlations do not show significant spatial decay even at higher temperatures where the correlations are small. Moreover the correlations in the doped case appear to be more robust against thermal fluctuations than in the undoped case. The characteristic temperature (determined from the half-width in Figs.8 and 10) is $T^{*} \sim 0.2 t$ for the 2-hole case while for the undoped system $T^{*} \sim 0.05 t$. This trend is consistent with the fact that in the doped case order is induced via the kinetic energy and the corresponding scale $t$ is larger than $J=0.25 t$. Although a more detailed analysis would be necessary to account properly for the doping dependence.

It has been shown recently by Ishihara et al. 28 that in the layered manganite compounds hydrostatic pressure leads to a stabilization of the $x^{2}-y^{2}$ orbitals as well.

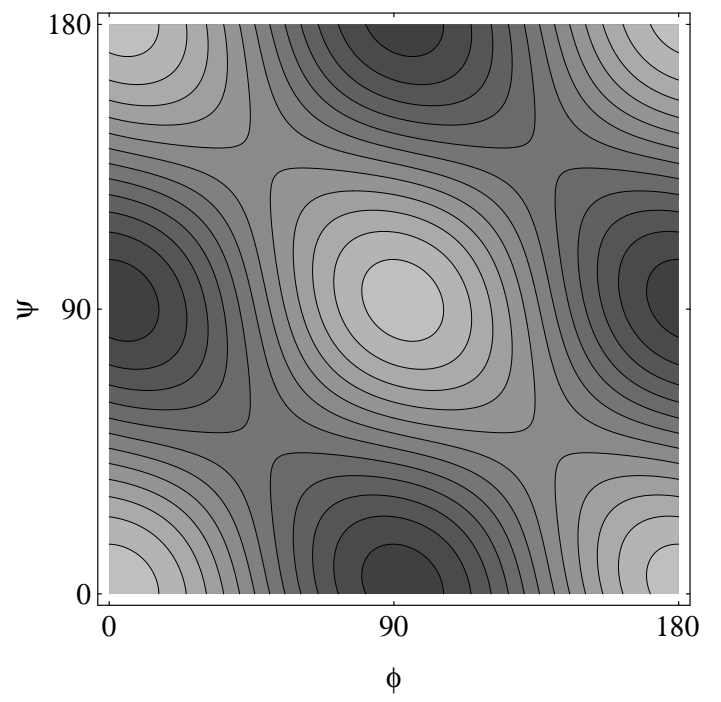

FIG. 9. Contourplot of $\left\langle\tilde{T}_{i}^{z} \tilde{T}_{i+R}^{z}\right\rangle$ for $R=(1,0)$ as function of angles $\phi$ and $\psi$ for a $\mathrm{N}=10$ site cluster with two holes. Otherwise same parameters as for Fig.7.

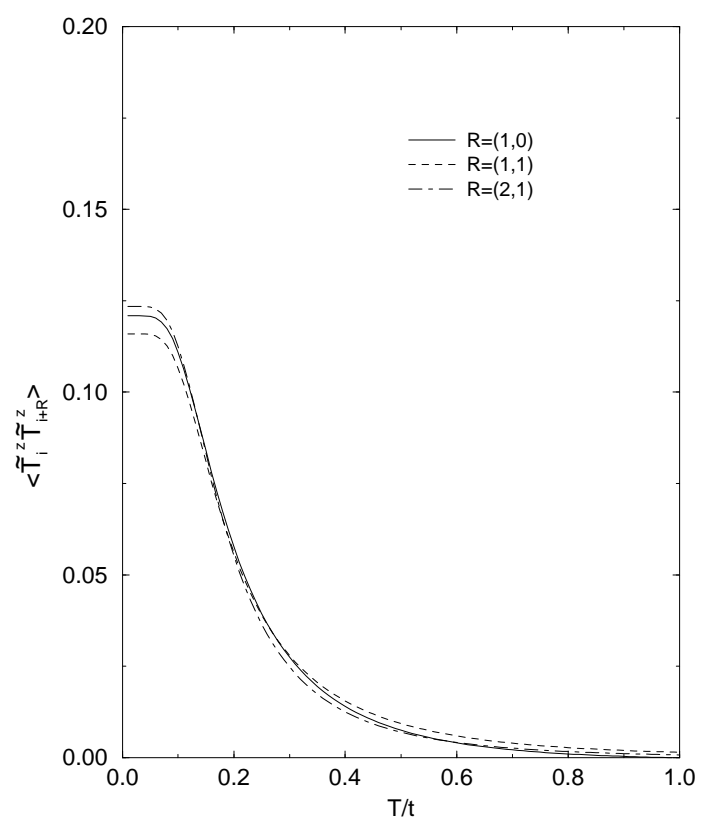

FIG. 10. Temperature dependence of $\left\langle\tilde{T}_{i}^{z} \tilde{T}_{i+R}^{z}\right\rangle$ with $\phi=0^{\circ}$ and $\psi=0^{\circ}$ for a 10 -site cluster with two $e_{g}$ holes. Parameters as in Fig.7. The strong increase below $T / t=0.3$ signals the onset of ferromagnetic orbital order in the planar model.

A comparison of the doping dependence of spatial correlations with the $t-J$ model is given in Fig.11 In the undoped case the orbital model shows almost classical (orbital) Néel order, whereas in the $t-J$ model correlations are strongly reduced by quantum fluctuations. The $T=0$ correlation function of the $t-J$ model shows longrange order (LRO) consistent with a strongly reduced sublattice magnetization $m_{z} \sim 0.3$ (in the thermodynamic limit). 


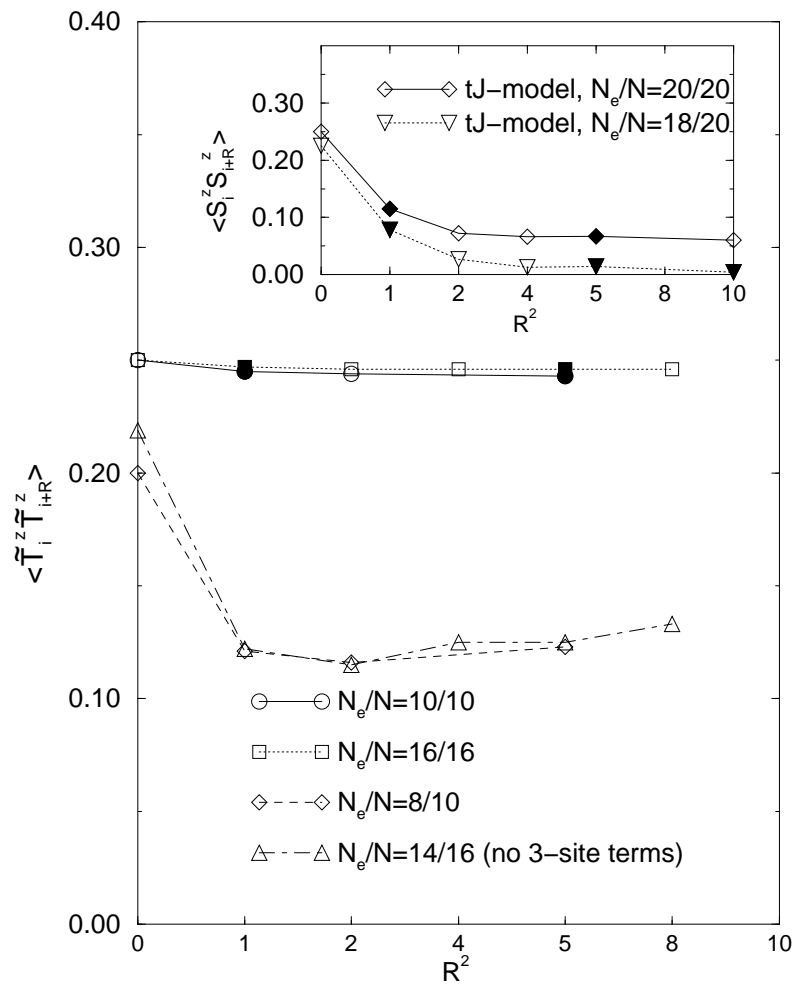

FIG. 11. Orbital correlation functions $(T \rightarrow 0)$ as function of distance (squared) for a 10-site and a $4 \times 4$ cluster with zero and two holes $(J / t=0.25)$. In the undoped case the correlation function is antiferromagnetic, whereas in the doped case orbital correlations are ferromagnetic. Antiferromagnetic orbital order is indicated by full (negative) and open (positive) symbols, respectively. Inset: The spin correlations $\left\langle S_{i}^{z} S_{i+R}^{z}\right\rangle$ for a 20 -site $2 \mathrm{D}$ Heisenberg model and the $t-J$ model with two holes are shown for comparison $(t-J$ data for $J=0.4$ from Ref.[47]). The two-hole case shows a rapid decay of correlations characteristic for an antiferromagnetic spin liquid.

In the orbital model the effect of the two holes $(x=$ 0.125 and 0.2$)$ is strong enough to induce ferromagnetic (orbital) correlations, i.e. with a prefered occupation of $x^{2}-y^{2}$ orbitals. In the $t-J$ model instead spin-correlations decay rapidly for two holes on a 20 -site cluster $(x=0.1)$, which is consistent with the notion of an $\mathrm{AF}$ spin-liquid state (See inset Fig.11).

The preference of ferromagnetic orbital order in the doped case is due to the fact that $t^{\alpha \beta}$ depends on the orbital orientation, and $t^{a a} \gg t^{b b}$. One would expect that for sufficiently strong orbital exchange interaction $J=4 t^{2} / U$ one reaches a point where antiferromagnetic orbital interactions and ferromagnetic correlations due to the kinetic energy are in balance. This quantum critical point between FM and AF orbital order turns out to be at quite large $J$ values. For the two-hole case $(x=0.2)$ we have found that this crossover happens for quite large orbital interaction $J \sim 2$, i.e. at a value about an order of magnitude larger than typical $J$ values in manganite systems.

$\mathrm{U} / \mathrm{t}$

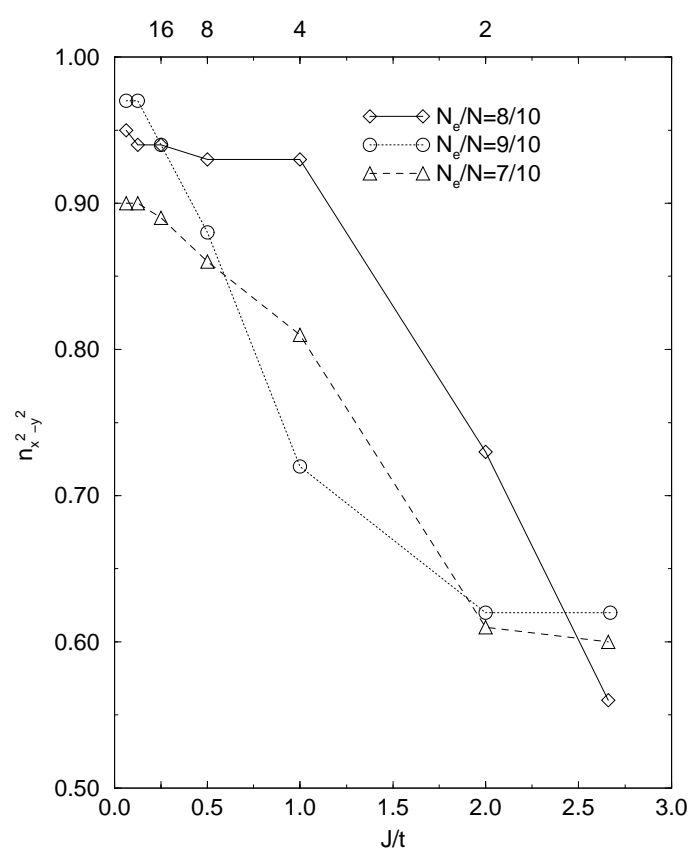

FIG. 12. Orbital occupation $n_{x^{2}-y^{2}}=N_{x^{2}-y^{2}} / N_{e}$ of the $x^{2}-y^{2}$ orbital as function of $J$ in doped systems for a 10 -site cluster with 1,2 , and 3 holes.

The relative $x^{2}-y^{2}$ orbital occupation is displayed in Fig. 12 for various doping concentrations as function of $J$ at low temperature. This clearly shows that for realistic values for $J$ a large occupation of the $x^{2}-y^{2}$ orbital is obtained. The predominance of this "ferromagnetic" orbital order distinguishes the charge propagation in the orbital model from the physics in the cuprates, where the carriers move in an antiferromagnetic spin liquid and at low doping in an antiferromagnetic ordered state, respectively. Nevertheless incoherent motion persists in the orbital model because of the non-diagonal hopping matrix elements $t^{\alpha \beta}$.

Before closing this section, we shall explain the different orbital occupancy in the doped and undoped case in more physical terms. The overlap of the two sets of orthogonal $e_{g}$ orbitals on neighboring sites changes with the angles $\phi$ and $\psi$. (1) In the undoped state the direct hopping between the (predominantely) occupied orbitals $\frac{1}{\sqrt{2}}(|x\rangle+|z\rangle)$ and $\frac{1}{\sqrt{2}}(|x\rangle-|z\rangle)$ on neighboring sites is small (Fig 13). The hopping between these occupied orbitals is blocked anyhow because of Pauli's principle. However $t^{a b}$ between an occupied orbital on one site and an unoccupied orbital on a neighbor site is extremal. This leads to a maximal antiferromagnetic orbital exchange interaction, and thereby to a lowering of the energy. (2) In the doped case instead, the $x^{2}-y^{2}$ orbital occupancy is prefered on all sites (Fig.14), which implies a large hop- 
ping amplitude for holes in the partially occupied band, whereas the off-diagonal hopping matrix element $t^{a b}$ and the orbital interaction are reduced for this choice of occupied orbitals. Hence the main energy gain in this case is due to the intraband correlated motion of holes in the $x^{2}-y^{2}$-band. Nevertheless the off-diagonal hopping plays an important role in the $x^{2}-y^{2}$ ordered state, and leads to the strong incoherent features characteristic for the conductivity $\sigma(\omega)$.

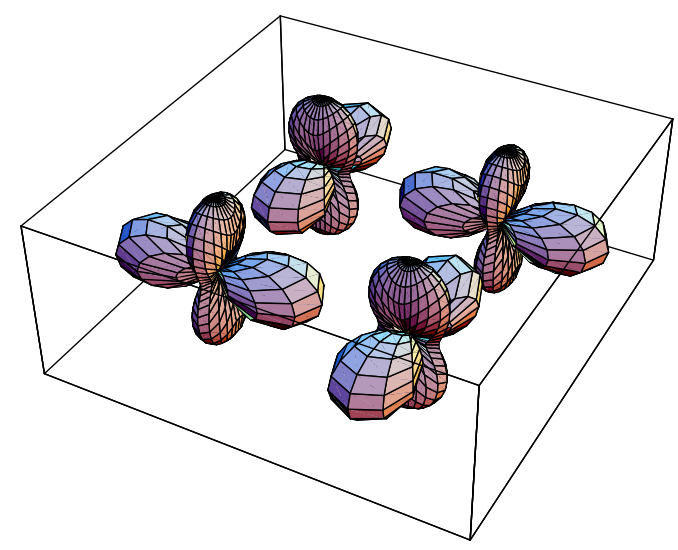

FIG. 13. Alternating $\frac{1}{\sqrt{2}}(|x\rangle+|z\rangle)$ and $\frac{1}{\sqrt{2}}(|x\rangle-|z\rangle)$ orbital order in the undoped planar system.

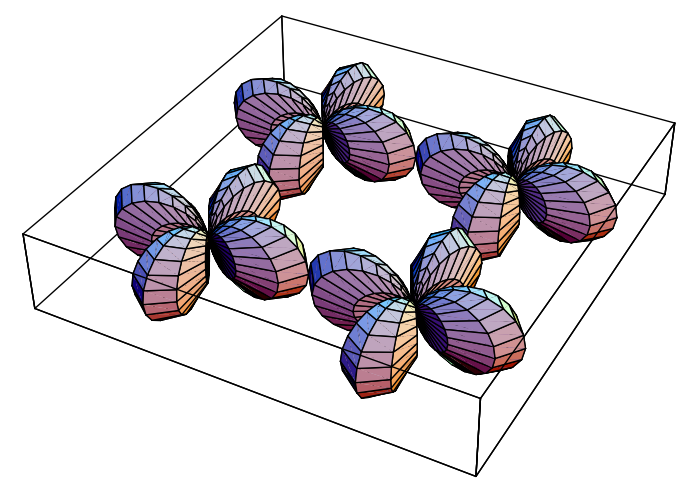

FIG. 14. $x^{2}-y^{2}$ orbital order in the doped phase.

Interestingly recent experiments by Akimoto et al. [48] proved the existence of an A-type antiferromagnetic metallic ground state in a wide doping concentration regime of the $3 \mathrm{D}$ system $\left(\mathrm{La}_{1-z} \mathrm{Nd}_{z}\right)_{1-x} \mathrm{Sr}_{x} \mathrm{MnO}_{3}$ and in particular for $\mathrm{La}_{1-x} \mathrm{Sr}_{x} \mathrm{Mn}_{3}$ at high doping $x=0.52-0.58$. The latter compound was investigated by Okimoto et al. for smaller doping $x$ where the ground state is the uniform ferromagnetic state. The A-type antiferromagnet consists out of ferromagnetic layers which are coupled antiferromagnetically. Akimoto et al. propose that $x^{2}-y^{2}$ orbitals should be occupied in this state and form a pseudo-2D band, as we found it here in our study of the ferromagnetic planar system. An experimental study of the optical properties in this concentration regime would be particularly interesting.

\section{SUMMARY AND CONCLUSIONS}

We have studied the optical conductivity, charge stiffness and the optical sum rule for an effective Hamiltonian that contains only the $e_{g}$-orbital degrees of freedom. This model follows from the more general Kondo lattice model, when the spins are ferromagnetically aligned. The 'orbital $t$ - $J$ model' is expected to describe the lowtemperature physics of manganese oxides in the ferromagnetic saturated phase.

This work was motivated by the experimental study of the frequency dependent conductivity of $\mathrm{La}_{1-x} \mathrm{Sr}_{x} \mathrm{MnO}_{3}$ by Tokura's group [3. In particular these experiments show in the saturated low- $T$ ferromagnetic phase a broad incoherent spectrum and at small frequency in addition a narrow Drude peak, which contains only about $20 \%$ of the total spectral weight. This experiment by itself shows that the ferromagnetic metallic state (although fully spin polarized) is highly anomalous. These features cannot be explained by the standard double exchange model, but require an additional mechanism. The most natural mechanism, one can think of, is the twofold degeneracy of the $e_{g}$ orbitals.

Our results for $\sigma(\omega)$ in the 2D orbital disordered regime show that these characteristic features follow from the orbital model. In particular there is (1) a broad continuum which decreases towards high frequency with a half-width $\omega_{1 / 2} \sim 2.5$ and $3.5 t$ in two and three dimensions, respectively. (2) The absorption increases with decreasing temperature and (3) the absolute value of the incoherent part of $\sigma(\omega)$ at low frequency and temperature $\sigma_{0}^{\text {inc }} \sim 0.4 \cdot 10^{3}(\Omega \mathrm{cm})^{-1}$ has the correct order of magnitude as in Tokura's experiments. (4) Despite this strong incoherence there is a finite Drude peak of about $20 \%$ of the total sum rule for small $J$. (5) The coherent motion and the Drude peak become more pronounced for larger values of $J$ due to the 3 -site hopping processes in the model. For the value $J / t=0.25$ estimated here the relative Drude weight is 40 (20) \% in the model with (without) 3-site hopping processes, respectively. (6) The low temperature values for the DC-conductivity and the resistivity can be estimated by assuming a width $\Gamma$ for the Drude peak (taken from experiment). The additional scattering processes contributing to $\Gamma$ are due to impurity or grain boundary scattering, i.e. these are extrinsic scattering processes which are not contained in the orbital model. The important information which follows 
from the model is the weight of the Drude peak, which together with $\Gamma$ determines the DC-conductivity.

The relatively small Drude peak in comparison with the incoherent part of $\sigma(\omega)$ clearly indicates that the carrier motion is essentially incoherent. Nevertheless we expect a quasiparticle peak in the single particle Green's function, yet with small spectral weight. Our study of the frequency dependent conductivity shows that the (saturated) ferromagnetic state in the manganites has unconventional transport properties due to the scattering from orbital excitations in combination with the exclusion of double occupancy. A detailed study of the orbital dynamics in the doped system is necessary for a deeper understanding of these issues.

The orbital model resembles the $t-J$ model, which describes the correlated motion of charge carriers in the cuprate superconductors. Yet an important difference is the cubic symmetry of the pseudospin representation of the orbital degrees of freedom. The hopping matrix elements depend on the orbital orientation, and are in general different in the two diagonal hopping channels. Moreover there is also an off-diagonal hopping matrix element, which does not exist at all in the $t$ - $J$ model. At low temperatures doping induces (ferromagnetic) $x^{2}-y^{2}$ orbital order for realistic values for the exchange interaction $J$ in the 2D orbital model. This is in striking contrast to $t$ - $J$ physics in cuprates, where the system changes upon doping from antiferromagnetic long-range order to an antiferromagnetic spin liquid. In both models coherent motion coexists with strong incoherent features.

Our calculations have shown that the orbital mechanism can explain the order of magnitude of the conductivity at low temperature. The orbital degrees of freedom are certainly also important for a quantitative calculation of the collossal magnetoresistance. This is obvious because in the full Kondo lattice model there is a close interplay between orbital and spin degrees of freedom 21,22.

\section{ACKNOWLEDGMENTS}

We enjoyed many stimulating discussions with J. van den Brink, G. Khaliullin and A. M. Oleś on the physics of the orbital model. Furthermore we acknowledge useful discussions with F. Assaad, L. Hedin, A. Muramatsu and R. Zeyher.

* Present address: Cadence Design Systems, D-85540 Haar, Germany

[1] A. J. Millis, Nature 392, 147 (1998).
[2] For a recent experimental review see: A. P. Ramirez, J. Phys.: Condens. Matter 9, 8171 (1997).

[3] Y. Okimoto, T. Katsufuji, T. Ishikawa, A. Urushibara, T. Arima and Y. Tokura, Phys. Rev. Lett 75109 (1995).

[4] Y. Okimoto, T. Katsufuji, T. Ishikawa, T. Arima, and Y. Tokura, Phys. Rev. B 55, 4206 (1997).

[5] S. G. Kaplan, U. M. Quijade, H. D. Drew, G. C. Xiong, R. Ramesh, C. Meuon, T. Venkatesan, and D. B. Tanner, Phys. Rev. Lett. 77, 2081 (1996).

[6] M. Quijada, J. Cerne, J. R. Simpson, H. D. Drew, K. H. Ahn, A. J. Millis, R. Shreekala, R. Ramesh, M. Rajeswari, and T. Venkatesan, cond-mat/9803201.

[7] K. H. Kim, J. H. Jung, and T. W. Noh, condmat/9804167, K. H. Kim et al., cond-mat/9804284.

[8] T. Ishikawa, T. Kimura, T. Katsufuji, and Y. Tokura, Phys. Rev. B 57, R8079 (1998) and references therein.

[9] N. Furukawa, J. Phys. Soc. Jpn. 64, 2734 (1995), ibid. 64, 3164 (1995).

[10] S. Ishihara, M. Yamanaka, and N. Nagaosa, Phys. Rev. B 56, 686 (1997).

[11] H. Shiba, R. Shiina, and A. Takahashi, J. Phys. Soc. Jpn. 66, 941 (1997).

[12] R. von Helmolt et al. Phys. Rev. Lett. 71, 2331 (1993); K. Chahara et al. Appl. Phys. Lett. 63, 1990 (1993); S. Jin et al. Science 264, 413 (1994); Y. Tokura et al. J. Phys. Soc. Jpn. 63, 3931 (1994).

[13] C. Zener, Phys. Rev. 82, 403 (1951); P. W. Anderson and H. Hasegawa, Phys. Rev. 100, 675 (1955); K. Kubo and A. Ohata, J. Phys. Soc Jpn 33, 21 (1972).

[14] A. J. Millis, P. B. Littlewood and B. I. Shraiman, Phys. Rev. Lett. 74, 5144 (1995); A. J. Millis, R. Mueller and B. I. Shraiman, Phys. Rev. B 54, 5405 (1996).

[15] J. Jaklič et al. (to be published).

[16] H. Röder, J. Zang, and A. R. Bishop, Phys. Rev. Lett. 76, 1356 (1996).

[17] K. J. von Szczepanski, P. Horsch, W. Stephan, and M. Ziegler, Phys. Rev. B 41, 2017 (1990).

[18] W. Stephan and P. Horsch, Int. J. Mod. Phys. B 6, 589 (1992); P. Horsch and W. Stephan, in The Hubbard Model, ed. by Dionys Baeriswyl et al. (Plenum, New York, 1995), p. 193.

[19] P. Horsch and W. Stephan, Phys. Rev. B 48, R 10595 (1993).

[20] H. Eskes, A. M. Oleś, M. Meinders, and W. Stephan, Phys. Rev. B 50, 17980 (1994).

[21] S. Ishihara, J. Inoue, and S. Maekawa, Phys. Rev. B 55, 8280 (1997).

[22] L. F. Feiner and A. M. Oleś, (preprint).

[23] J. Jaklič and P. Prelovšek, Phys. Rev. B 49, 5065 (1994); ibid 50, 7129 (1994); ibid 52, 6903 (1995).

[24] For a review see J. Jaklič and P. Prelovšek, condmat/9803331.

[25] J. C. Slater and G. F. Koster, Phys. Rev. 94, 1498 (1954).

[26] K. I. Kugel and D. I. Khomskii, Sov. Phys. JETP 37, 725 (1973).

[27] W. A. Harrison, Electronic Structure and Properties of Solids, (Freeman, San Francisco, 1980).

[28] S. Ishihara, S. Okamoto, and S. Maekawa, condmat/9712162.

[29] J. Zaanen and A. M. Oleś, Phys. Rev. B 48, 7197 (1993).

[30] C. M. Varma, Phys. Rev. B 54, 7328 (1996). 
[31] A. H. MacDonald, S. M. Girvin, and D. Yoshioka, Phys. Rev. B 37, 9753 (1988) and references therein.

[32] B. S. Shastry and B. Sutherland, Phys. Rev. Lett. 65, 243 (1990).

[33] A. Urushibara et al., Phys. Rev. B 51, 14103 (1995).

[34] W. Kohn, Phys. Rev. 133, A171 (1964).

[35] D. Baeriswyl, J. Carmelo, and A. Luther, Phys. Rev. B 16, 7247 (1986).

[36] The same twisted boundary conditions were used for the finite temperature studies in this work. These boundary conditions guarantee a nondegenerate closed-shell ground state.

[37] F. Mack and P. Horsch, unpublished.

[38] D. Poilblanc and E. Dagotto, Phys, Rev. B 44, 466 (1991).

[39] B. F. Woodfield, M. L. Wilson, and J. M. Byers, Phys. Rev. Lett. 78, 3201 (1997).

[40] R. Kilian and G. Khaliullin, preprint.

[41] J. B. Goodenough, Phys. Rev. 100, 564 (1955).

[42] J. B. Goodenough, in Progress in Solid State Chemistry, Vol.5, ed. by H. Reiss (Pergamon, London, 1971).

[43] E. D. Wollan and W. C. Koehler, Phys. Rev. 100, 545 (1955).

[44] G. Matsumoto, J. Phys. Soc. Jpn. 29, 606 (1970).

[45] J. B. A. A. Elemans, B. van Laar, K. R. van der Veen, and B. O. Loopstra, J. Solid State Chem. 3, 238 (1971).

[46] This particular orbital order for the undoped 2D orbital model was found independently by J. van den Brink et al. (unpublished).

[47] P. Horsch and W. Stephan, Physica C 185-189, 1585 (1991).

[48] T. Akimoto, Y. Maruyama, Y. Moritomo, A. Nakamuram K. Hirota, K. Ohoyama, and M. Ohashi, Phys. Rev. B 57, R5594 (1998). 